\title{
A PROPERTY OF POLYNOMIAL CURVES OVER A FIELD OF POSITIVE CHARACTERISTIC
}

\author{
D. DAIGLE
}

(Communicated by Louis J. Ratliff, Jr.)

\begin{abstract}
Let $\mathbf{k}$ be an algebraically closed field of characteristic $p>0$. We show that if $F \in \mathbf{k}[X, Y]$ is a rational curve with one place at infinity and with nonprincipal bidegree, such that $\theta(F) \in \mathbf{k}\left[X^{p}, Y\right]$ for some automorphism $\theta$ of $\mathbf{k}[X, Y]$, then $\theta$ can be chosen to be either linear or of "de Jonquière" type. We also give consequences of that fact for the problem of classifying the embeddings of the line in the plane.
\end{abstract}

Let $\mathbf{k}$ be an algebraically closed field of characteristic $p>0, X, Y, T$ indeterminates over $\mathbf{k}, R=\mathbf{k}[X, Y], R_{0}=\mathbf{k}\left[X^{p}, Y\right]$ and $\mathbf{k}^{*}=\mathbf{k} \backslash\{0\}$. Let Aut $_{\mathbf{k}} R$ be the group of $\mathbf{k}$-algebra automorphisms of $R$ and say that $F, G \in R$ are equivalent $(F \sim G)$ if $G=\lambda \theta(F)$ for some $\theta \in$ Aut $_{\mathbf{k}} R$ and $\lambda \in \mathbf{k}^{*}$.

A polynomial curve is an irreducible $F \in R$ such that $F(x, y)=0$ for some $x, y \in \mathbf{k}[T]$ with $\mathbf{k}(x, y)=\mathbf{k}(T)$. Such a pair $(x, y)$ is then called a parametrization of $F$. The key result of this paper is

Theorem. Suppose $F$ is a polynomial curve with $0<\operatorname{deg}_{Y} F<\operatorname{deg}_{X} F$ and $\operatorname{deg}_{Y} F \nmid \operatorname{deg}_{X} F$. If $F$ is equivalent to an element of $R_{0}$ then either $F(Y, X) \in$ $R_{0}$ or $F(X, Y+f(X)) \in R_{0}$, for some $f(X) \in \mathbf{k}[X]$ with $\operatorname{deg} f(X) \operatorname{deg}_{Y} F<$ $\operatorname{deg}_{X} F+\operatorname{deg}_{Y} F$.

It is interesting to note that this theorem does not generalize to plane curves with one place at infinity-see (1.8). Also, the theorem has interesting consequences for the problem of classifying the embeddings of the line in the affine plane, as will be discussed in the second section.

\section{Polynomial CURves}

1.1. We begin by recalling some notations and definitions. We also state some basic facts concerning plane curves with one place at infinity; these facts can be either found in or deduced from [1].

Received by the editors June 2, 1989 and, in revised form, on October 2, 1989.

1980 Mathematics Subject Classification (1985 Revision). Primary 14E25, 13 F20.

Partly supported by a fellowship from NSERC Canada. 
1. Given $x \in \mathbf{k}[T]$, the formal derivative $d x / d T$ is denoted $\dot{x}$.

2. Let $F \in R$. The formal derivatives $\partial F / \partial X$ and $\partial F / \partial Y$ are denoted $F_{X}$ and $F_{Y}$ respectively. If $F=\sum a_{i j} X^{i} Y^{j} \quad\left(a_{i j} \in \mathbf{k}\right)$ then $F^{(p)}=$ $\sum a_{i j}^{p} X^{i} Y^{j}$. If $F \neq 0$, let $\operatorname{deg}_{X} F=\max \left\{i \mid a_{i j} \neq 0\right.$ for some $\left.j\right\}$, $\operatorname{deg}_{Y} F=\max \left\{j \mid a_{i j} \neq 0\right.$ for some $\left.i\right\}$; also define $\operatorname{deg}_{X} 0=\operatorname{deg}_{Y} 0=0$ and $\operatorname{deg} 0=0$. The bidegree of $F$ is bideg $F=\left(\operatorname{deg}_{X} F, \operatorname{deg}_{Y} F\right)$.

3. To compare bidegrees, we partially order $\mathbf{Z}^{2}$ (where $\mathbf{Z}$ is the ring of integers) by declaring $(a, b)<(c, d)$ if either $\min (a, b)<\min (c, d)$, or $\min (a, b)=\min (c, d)$ and $\max (a, b)<\max (c, d)$.

4. $(a, b) \in \mathbf{Z}^{2}$ is said to be nonprincipal if either $a b=0$, or $a \nmid b$ and $b \nmid a . F \in R$ has nonprincipal bidegree if bideg $F$ is nonprincipal.

5. An integral domain which strictly contains $\mathbf{k}$ is said to have one place at infinity if it is contained in all but one of the valuation rings (containing k) of its field of fractions. $F \in R$ is said to have one place at infinity if it is irreducible and $R / F R$ has one place at infinity.

6. Suppose $F \in R$ has one place at infinity, $\operatorname{deg}_{X} F=n>0$ and $\operatorname{deg}_{Y} F=m>0$.

- If $m \mid n$, say $m d=n$, then $\operatorname{bideg} F\left(X, Y+\lambda X^{d}\right)<\operatorname{bideg} F$ for a unique $\lambda \in \mathbf{k}^{*}$.

- If $n \mid m$, say $n d=m$, then $\operatorname{bideg} F\left(X+\lambda Y^{d}, Y\right)<\operatorname{bideg} F$ for a unique $\lambda \in \mathbf{k}^{*}$.

In particular, $F$ is equivalent to some $G$ with nonprincipal bidegree.

7. We define an equivalence relation $\simeq$ on $R$ by declaring $F \simeq G$ if there are $\lambda, a, c \in \mathbf{k}^{*}$ and $b, d \in \mathbf{k}$ such that $G=\lambda F(a X+b, c Y+d)$. Note that if $F \simeq G$ then $F_{X}=0 \Leftrightarrow G_{X}=0$ and $F_{Y}=0 \Leftrightarrow G_{Y}=0$.

8. Suppose $F \in R$ (resp. $G \in R$ ) has one place at infinity, has nonprincipal bidegree and satisfies $\operatorname{deg}_{Y} F<\operatorname{deg}_{X} F\left(\right.$ resp. $\left.\operatorname{deg}_{Y} G<\operatorname{deg}_{X} G\right)$. If $F \sim G$ then bideg $F=\operatorname{bideg} G$ and $G \simeq F(X, Y+f(X))$ for some $f(X) \in \mathbf{k}[X]$ with $\operatorname{deg} f(X) \operatorname{deg}_{Y} F<\operatorname{deg}_{X} F$.

9. For $F \in R$, the following are equivalent:

- $F$ is a polynomial curve,

- $F$ has one place at infinity and has genus zero (which means that the field of fractions of $R / F R$ is isomorphic to $\mathbf{k}(T))$.

10. If $(x, y)$ is a parametrization of a polynomial curve $F$ then $\operatorname{deg}_{X} F=$ $\operatorname{deg} y$ and $\operatorname{deg}_{Y} F=\operatorname{deg} x$. Moreover, $F \in R_{0} \Leftrightarrow y \in \mathbf{k}\left[T^{p}\right]$ and $F \in \mathbf{k}\left[X, Y^{p}\right] \Leftrightarrow x \in \mathbf{k}\left[T^{p}\right]$.

Definition 1.2. We define an injective ring homomorphism $\varphi: R \rightarrow R$ by $\varphi(F)=F^{(p)}\left(X^{p}, Y\right)$. Note that $\varphi(R)=R_{0}$.

1.3. Let $F \in R$ be such that $F_{Y} \neq 0$. Observe that

- $F$ is irreducible if and only if $\varphi(F)$ is.

- If $F$ is irreducible and $x, y$ are the images of $X, Y$ in $R / F R$, then $\mathbf{k}\left(x, y^{p}\right)=\mathbf{k}(x, y)$. Moreover, $\varphi(F)\left(x, y^{p}\right)=0$, which allows us to identify $R / \varphi(F) R$ with the subring $\mathbf{k}\left[x, y^{p}\right]$ of $\mathbf{k}[x, y]=R / F R$. 
- $F$ has one place at infinity if and only if $\varphi(F)$ has one place at infinity.

- $F$ is a polynomial curve if and only if $\varphi(F)$ is a polynomial curve.

\section{Definitions 1.4.}

1. An element $\theta$ of Aut $_{\mathbf{k}} R$ is called a $\varphi$-automorphism of $R$ if it satisfies the following equivalent conditions:

- There exists $\theta^{\prime} \in \mathrm{Aut}_{\mathbf{k}} R$ such that $\theta \varphi=\varphi \theta^{\prime}$,

- $\theta\left(R_{0}\right)=R_{0}$,

- $\theta(Y) \in R_{0}$.

The set of all $\varphi$-automorphisms of $R$ is a subgroup of Aut $_{\mathbf{k}} R$ denoted Aut $_{\varphi} R$.

2. $F, G \in R$ are $\varphi$-equivalent $(F \approx G)$ if $G=\lambda \theta(F)$ for some $\theta \in$ Aut $_{\varphi} R$ and $\lambda \in \mathbf{k}^{*}$. Note that if $F \approx G$ then $F \in R_{0} \Leftrightarrow G \in R_{0}$.

3. Let $F \in R$ have one place at infinity. Say that $F$ is $\varphi$-stopped if the following hold:

- $\operatorname{deg}_{X} F \nmid \operatorname{deg}_{Y} F$,

- $p \operatorname{deg}_{Y} F \nmid \operatorname{deg}_{X} F$,

- $\operatorname{deg}_{Y} F \mid \operatorname{deg}_{X} F$.

Note that if $F$ is $\varphi$-stopped then it does not have nonprincipal bidegree.

Lemma 1.5. If $F \in R$ has one place at infinity then $F \approx G$ for some $G$ which either is $\varphi$-stopped or has nonprincipal bidegree.

Proof. Suppose $F$ is not $\varphi$-stopped and does not have nonprincipal bidegree. Write bideg $F=(n, m)$; then $m n \neq 0$ and either $n \mid m$ or $p m \mid n$. In the first case, let $d=m / n$ and $H=F\left(X+\lambda Y^{d}, Y\right)$; in the second case, let $d=n /(p m)$ and $H=F\left(X, Y+\lambda X^{p d}\right)$. Since $F \approx H$ and since, by (1.1.6), in each case $\lambda \in \mathbf{k}^{*}$ may be chosen so that bideg $H<\operatorname{bideg} F$, we are done.

Lemma 1.6. Let $F_{1}, F_{2} \in R$ and suppose that $F_{2} \simeq F_{1}\left(X, Y+f_{1}(X)\right)$ for some $f_{1}(X) \in \mathbf{k}[X]$. Then $F_{1} \simeq F_{2}\left(X, Y+f_{2}(X)\right)$ for some $f_{2}(X) \in \mathbf{k}[X]$ with $\operatorname{deg} f_{2}(X)=\operatorname{deg} f_{1}(X)$.

Proof. Write $\lambda F_{2}(a X+b, c Y+d)=F_{1}\left(X, Y+f_{1}(X)\right)$, where $\lambda, a, c \in$ $\mathbf{k}^{*}$ and $b, d \in \mathbf{k}$. Substituting $Y-f_{1}(X)$ for $Y$ in that equation, we get $F_{1}=\lambda F_{2}\left(a X+b, c Y-c f_{1}(X)+d\right)$. Substituting $a^{-1} X-a^{-1} b$ for $X$ and $c^{-1} Y-c^{-1} d$ for $Y$ yields

$$
F_{1}\left(a^{-1} X-a^{-1} b, c^{-1} Y-c^{-1} d\right)=\lambda F_{2}\left(X, Y-c f_{1}\left(a^{-1} X-a^{-1} b\right)\right),
$$

hence $F_{1} \simeq F_{2}\left(X, Y+f_{2}(X)\right)$ with $f_{2}(X)=-c f_{1}\left(a^{-1} X-a^{-1} b\right)$.

Proof of the main theorem. We may assume that $F \notin \mathbf{k}\left[X, Y^{p}\right]$, i.e., $F_{Y} \neq 0$. By applying (1.5) to an element of $R_{0}$ equivalent to $F$, we see that $F$ is equivalent to some $G \in R_{0}$ such that $G$ has nonprincipal bidegree or is $\varphi$ stopped. 
If $G$ has nonprincipal bidegree then, by (1.1.8), there exists $f(X) \in \mathbf{k}[X]$ with $\operatorname{deg} f(X) \operatorname{deg}_{Y} F<\operatorname{deg}_{X} F$ such that if we set $H=F(X, Y+f(X))$ then $H \simeq G$ or $H \simeq G(Y, X)$. Since $F_{Y} \neq 0$, we have $H_{Y} \neq 0$, thus $H \not G(Y, X)$. Therefore $H \simeq G \in R_{0}$, whence $H \in R_{0}$ and we are done.

If $G$ is $\varphi$-stopped, write bideg $G=(n, m)$. Then it follows from (1.1.6) that there exists $g(X) \in \mathbf{k}[X]$ of degree $d=n / m$ such that $H=G(X, Y+g(X))$ satisfies $\operatorname{deg}_{Y} H \nmid \operatorname{deg}_{X} H$. Note that $d \geq 2$ and $d \not \equiv 0(\bmod p)$.

Claim. $0<\operatorname{deg}_{Y} H<\operatorname{deg}_{X} H$. Clearly, $\operatorname{deg}_{Y} H=\operatorname{deg}_{Y} G>0$. For the other inequality, consider a parametrization $(x, y)$ of $G$; then $\operatorname{deg} x=m, \dot{y}=0$ and $(x, y-g(x))$ is a parametrization of $H$. Now

$$
\operatorname{deg}_{X} H=\operatorname{deg}(y-g(x))>\operatorname{deg} \frac{d}{d T}(y-g(x))=\operatorname{deg}\left(g^{\prime}(x) \dot{x}\right) \geq \operatorname{deg} g^{\prime}(x)
$$

since $\dot{x} \neq 0$. Since $\operatorname{deg} g^{\prime}(x)=(d-1) m=(d-1) \operatorname{deg}_{Y} H$ we have in fact

$$
\operatorname{deg}_{X} H>(d-1) \operatorname{deg}_{Y} H,
$$

from which the claim follows. In particular, $H$ has nonprincipal bidegree. By (1.1.8), it follows that bideg $F=\operatorname{bideg} H$ and $F \simeq H(X, Y+h(X))$, for some $h(X) \in \mathbf{k}[X]$ such that

$$
\operatorname{deg} h(X)<\frac{\operatorname{deg}_{X} H}{\operatorname{deg}_{Y} H}=\frac{\operatorname{deg}_{X} H}{\operatorname{deg}_{Y} G}<\frac{\operatorname{deg}_{X} G}{\operatorname{deg}_{Y} G}=d .
$$

So $\operatorname{deg}(g(X)+h(X))=d$ and $F \simeq G(X, Y+g(X)+h(X))$. By the first part of (1.6), there exists $f(X) \in \mathbf{k}[X]$ of degree $d$ such that $F(X, Y+f(X)) \simeq$ $G \in R_{0}$, hence $F(X, Y+f(X)) \in R_{0}$. Since $\operatorname{deg}_{X} H>(d-1) \operatorname{deg}_{Y} H$ and $\operatorname{bideg} F=\operatorname{bideg} H$ we see that $\operatorname{deg} f(X)$ satisfies the desired condition, and this completes the proof.

Note that the following fact was established in the above proof.

Lemma 1.7. Suppose $G$ is a polynomial curve, $G$ is $\varphi$-stopped and $G \in R_{0}$. Then, for some $g(X) \in \mathbf{k}[X]$, the polynomial $H=G(X, Y+g(X))$ satisfies

$$
0<\operatorname{deg}_{Y} H<\operatorname{deg}_{X} H \text { and } \operatorname{deg}_{Y} H \nmid \operatorname{deg}_{X} H \text {. }
$$

Moreover, the degree $d$ of any such $g(X)$ satisfies $d=\operatorname{deg}_{X} G / \operatorname{deg}_{Y} G, d \geq 2$ and $d \not \equiv 0(\bmod p)$.

As mentioned in the introduction, the theorem does not generalize to all $F \in R$ with one place at infinity. This is shown in

Example 1.8. Let char $\mathbf{k}=3$; then $F=-X^{5}-X^{4}-X^{3} Y+X^{2} Y+X+Y^{3}-Y^{2}$ has one place at infinity but is not a polynomial curve (one can see that the genus is 4). It satisfies $0<\operatorname{deg}_{Y} F<\operatorname{deg}_{X} F$ and $\operatorname{deg}_{Y} F+\operatorname{deg}_{X} F$. We have $F \sim F_{1} \sim F_{2} \sim F_{3} \in R_{0}$, where

$$
\begin{aligned}
& F_{1}=F\left(X, Y+X^{3}-X^{2}\right)=X^{9}+X+Y^{3}-Y^{2}, \\
& F_{2}=F_{1}\left(X+Y^{2}, Y\right)=X^{9}+X+Y^{18}+Y^{3}, \\
& F_{3}=F_{2}(Y, X)=X^{18}+X^{3}+Y^{9}+Y .
\end{aligned}
$$


On the other hand, $F(Y, X) \notin R_{0}$ and if, for some $f(X) \in \mathbf{k}[X]$, the polynomial $G=F(X, Y+f(X))$ is in $R_{0}$, then

$$
0=G_{X}=F_{X}(X, Y+f(X))+F_{Y}(X, Y+f(X)) f^{\prime}(X) .
$$

Looking at $G_{X}$ as a polynomial in $Y$, we see that the coefficient of $Y$ is $f^{\prime}(X)-X$. Hence $f^{\prime}(X)=X$ and the above equation becomes

$$
0=F_{X}(X, Y+f(X))+F_{Y}(X, Y+f(X)) X=1,
$$

which is absurd. Hence the theorem does not hold for $F$.

One can prove that, for the $F$ given in (1.8), bideg $G>\operatorname{bideg} F$ for all $G$ such that $\varphi(G) \sim F$. Hence the next result cannot be generalized either.

Corollary 1.9. Let $F \in R$ be a polynomial curve not equivalent to $Y$. If there exists $G \in R$ such that $F \sim \varphi(G)$, then there exists $H \in R$ such that $F \sim \varphi(H)$ and bideg $H<\operatorname{bideg} F$.

Proof. We first consider the case where $F$ has nonprincipal bidegree. Replacing, if necessary, $F$ by $F(Y, X)$, we may assume that $0<\operatorname{deg}_{Y} F<$ $\operatorname{deg}_{X} F$ (we have $\operatorname{deg}_{Y} F>0$ because $F \nsim Y$ ). By the main theorem, either $F(Y, X) \in R_{0}$ or $F(X, Y+f(X)) \in R_{0}$ for some $f(X) \in \mathbf{k}[X]$ with $\operatorname{deg} f(X) \operatorname{deg}_{Y} F<\operatorname{deg}_{X} F+\operatorname{deg}_{Y} F$.

In the first case, write $F(Y, X)=\varphi(H)$; then clearly bideg $H<\operatorname{bideg} F$.

In the second case, write $F(X, Y+f(X))=\varphi(H)$. So $\operatorname{deg}_{Y} H=\operatorname{deg}_{Y} \varphi(H)$ $=\operatorname{deg}_{Y} F$ and, by the condition on $\operatorname{deg} f(X)$,

$$
\operatorname{deg}_{X} H=\frac{1}{p} \operatorname{deg}_{X} \varphi(H)<\operatorname{deg}_{X} F,
$$

i.e., bideg $H<\operatorname{bideg} F$.

If $F$ does not have nonprincipal bidegree, there exists $F_{0} \sim F$ such that $F_{0}$ has nonprincipal bidegree and $\operatorname{bideg} F_{0}<\operatorname{bideg} F$. The result follows by applying the case proved above to $F_{0}$.

Lemma 1.10. If $\theta \in \mathrm{Aut}_{k} R$ satisfies $R_{0} \cap \theta^{-1}\left(R_{0}\right) \notin R^{p}$ then $\theta \in \operatorname{Aut}_{\varphi} R$. In particular, for $F, G \in R_{0} \backslash R^{p}$ we have $F \sim G \Rightarrow F \approx G$.

Proof. Choose $F \in R_{0} \cap \theta^{-1}\left(R_{0}\right)$ with $F \notin R^{p}$. Then $F_{X}=0, F_{Y} \neq 0$, and $\theta(F) \in R_{0}$, so

$$
0=\frac{\partial}{\partial X}(\theta(F))=\frac{\partial}{\partial X} F(\theta(X), \theta(Y))=F_{Y}(\theta(X), \theta(Y)) \frac{\partial}{\partial X}(\theta(Y)),
$$

which implies that $\frac{\partial}{\partial X}(\theta(Y))=0$, and hence that $\theta \in \operatorname{Aut}_{\varphi} R$. The last assertion is clear.

Corollary 1.11. Suppose $F, G \in R$ are such that $F_{Y} \neq 0$ and $G_{Y} \neq 0$. If $\varphi(F) \sim \varphi(G)$ then $F \sim G$.

Proof. Observe that, for $F, G \in R, \varphi(F) \approx \varphi(G) \Rightarrow F \sim G$. Hence this follows immediately from (1.10).

Remark. $F \sim G \nRightarrow \varphi(F) \sim \varphi(G)$. 


\section{LINES}

The results of the first section have interesting consequences for the classification of lines problem. We now recall that problem (see $[2,3,5,6])$.

A line is an $F \in R$ such that $R / F R \cong \mathbf{k}[T]$; hence, in particular, a line is a polynomial curve. Two lines $F_{1}, F_{2}$ are equivalent if $F_{1} \sim F_{2}$ as defined in the introduction. It can be seen that the set of equivalence classes of lines has cardinality $|\mathbf{k}|$ (in contrast with the situation in characteristic zero, where all lines are equivalent-see [2]). A line $F$ is a coordinate line if $F \sim Y$; otherwise it is said to be wild. Every example of wild line currently known, say $F$, can be obtained as $F=F_{N}$ for some sequence of lines $\left(F_{0}, \ldots, F_{N}\right)$ such that $F_{0}=Y$ and, for each $i$ with $0 \leq i<N$, either $F_{i+1} \sim F_{i}$ or $F_{i+1}=\varphi\left(F_{i}\right)$. (Note that, for $F \in R, \varphi(F)$ is a line $\Leftrightarrow F$ is a line and $F_{Y} \in \mathbf{k}^{*}$. Equivalently, if $x$, $y \in \mathbf{k}[T]$ then $\mathbf{k}\left[x, y^{p}\right]=\mathbf{k}[T] \Leftrightarrow \mathbf{k}[x, y]=\mathbf{k}[T]$ and $\dot{x} \in \mathbf{k}^{*}$. See Proposition 1 of [6] for this.)

Let us consider an oriented graph $\mathscr{L}$. whose vertices are the equivalence classes of lines and whose links are defined as follows:

- There is no link from a vertex to itself.

- If $A$ and $B$ are distinct vertices then there is at most one link going from $A$ to $B$, and there is a link if and only if there exists $F \in A$ such that $\varphi(F) \in B$.

We write $A \rightarrow B$ to indicate that there is a link from $A$ to $B$. If $C_{0}$ denotes the vertex containing the coordinate lines, then the fact stated above says that if $A$ is any vertex of $\mathscr{L}$ currently known then there exists a path $C_{0}=A_{0} \rightarrow \cdots \rightarrow A_{n}=A$. It is conjectured that all vertices have this property. As far as we know, the strongest version of that conjecture is Conjecture 1 of [6], which can be stated as

Conjecture 1. If $F \in R$ is a wild line then there exists $\theta \in \mathrm{Aut}_{\mathrm{k}} R$ such that

1. $\theta(F) \in \mathbf{k}\left[X^{p}, Y\right]$, i.e., $\theta(F)=\varphi(G)$ for some line $G$;

2. bideg $G<\operatorname{bideg} F$.

A seemingly weaker version is obtained by dropping the second requirement:

Conjecture 2. If $F \in R$ is a line then there exists $\theta \in \mathrm{Aut}_{\mathbf{k}} R$ such that $\theta(F) \in$ $\mathbf{k}\left[X^{p}, Y\right]$.

However, (1.9) shows that the second conjecture is in fact equivalent to the first one.

We will now derive some properties of $\mathscr{L}$ from the results of the first section. First, observe that $\mathscr{L}$ is a subgraph of the oriented graph $\mathscr{P}$ whose vertices are the equivalence classes of polynomial curves, and whose links are defined exactly as in the case of $\mathscr{L}$. Further, define a partial order on the set of vertices of $\mathscr{P}$ by declaring $A<B$ if there exists $F_{0} \in A$ such that $\operatorname{bideg} F_{0}<\operatorname{bideg} F$ for all $F \in B$. Then it is clear that $C_{0}<B$ whenever $B$ is any vertex of $\mathscr{P}$ other than $C_{0}$. Also, note that $C_{0}$ is not the target of any link; indeed, if $F$ 
is a polynomial curve and $\varphi(F) \in C_{0}$ then $\varphi(F) \sim Y=\varphi(Y) \Rightarrow F \sim Y$ by (1.11). Some corollaries of the results of the first section are gathered in

\section{Theorem 2.1.}

1. No vertex of $\mathscr{P}$ is the target of more than one link.

2. For distinct vertices $A$ and $B$ of $\mathscr{P}$, we have $A<B$ whenever $A \rightarrow B$.

3. No sequence $\left(A_{i}\right)_{i=1}^{\infty}$ of vertices of $\mathscr{P}$ satisfies $A_{i} \leftarrow A_{i+1}$ for all $i$.

4. $\mathscr{P}$ has no loops.

5. Each connected component of $\mathscr{P}$ contains exactly one vertex which is not the target of any link.

Proof. (1) is just (1.11). For (2), consider a link $A \rightarrow B$. Choose $F \in B$ with nonprincipal bidegree; for some $G \in A$ we have $\varphi(G) \in B$, hence $F \sim \varphi(G)$. Since $B$ is a target we have $B \neq C_{0}$, hence $F \nsim Y$ and by (1.9) $F \sim \varphi(H)$ for some $H$ such that bideg $H<\operatorname{bideg} F$; by (1.11), $H \in A$. Clearly, bideg $H<$ bideg $F^{\prime}$. for all $F^{\prime} \in B$ and (2) is proved.

To prove (3), observe that such a sequence $\left(A_{i}\right)_{i=1}^{\infty}$ would give rise, by (2), to an infinite sequence

$$
\left(a_{1}, b_{1}\right)>\left(a_{2}, b_{2}\right)>\cdots
$$

in $\mathbf{Z}^{2}$, with $\left(a_{i}, b_{i}\right)>(0,0)$ for all $i$. Now, such a sequence does not exist.

If there is a loop in $\mathscr{P}$, then that loop either contains a vertex which is the target of two links, or gives rise to a sequence $\left(A_{i}\right)_{i=1}^{\infty}$ of vertices such that $A_{i} \leftarrow A_{i+1}$ for all $i$. So (4) follows from (1) and (3).

By (3), each connected component of $\mathscr{P}$ contains at least one vertex which is not the target of any link; uniqueness follows from (1), so (5) is proved.

Clearly, this theorem is still true if we replace " $\mathscr{P}$ " by " $\mathscr{L}$ " everywhere. (R. Ganong pointed out that the third assertion of the theorem (for $\mathscr{L}$ ) can also be deduced from [4, Theorem 1.4], first assertion.) So, in particular, from the last assertion of the theorem we see that the conjectures are equivalent to connectedness of $\mathscr{L}$. We also point out that the main theorem gives us a lot of information about the conjectured automorphism.

More generally, one may dream of classifying the polynomial curves, i.e., understanding which vertices of $\mathscr{P}$ are not targets of links. For this, too, the main theorem gives interesting information. For instance, let $m, n$ be relatively prime integers such that $1<m<n$ and $m n \not \equiv 0(\bmod p)$, and let $C_{m, n}$ be the vertex of $\mathscr{P}$ which contains $X^{n}+Y^{m}$. Then it follows from the main theorem that $C_{m, n}$ is not a target. Hence, by (2.1), $\mathscr{P}$ has infinitely many connected components.

To conclude, we note that the main theorem can be stated in terms of parametrizations as follows. 
Corollary 2.2. Let $F$ be a polynomial curve with parametrization $(x, y)$ such that $0<\operatorname{deg} x<\operatorname{deg} y$ and $\operatorname{deg} x \nmid \operatorname{deg} y$. If there exists $\theta \in \mathrm{Aut}_{\mathbf{k}} R$ such that $\theta(F) \in \mathbf{k}\left[X^{p}, Y\right]$ then one of the following conditions holds:

1. $x \in \mathbf{k}\left[T^{p}\right]$,

2. $y-f(x) \in \mathbf{k}\left[T^{p}\right]$ for some $f(X) \in \mathbf{k}[X]$ with $\operatorname{deg} f(X) \operatorname{deg} x<\operatorname{deg} x+$ $\operatorname{deg} y$.

\section{ACKNOWLEDGMENT}

I thank A. Sathaye for his inexhaustible willingness to discuss polynomials in two variables.

\section{REFERENCES}

1. S. Abhyankar, Lectures on expansion techniques in algebraic geometry (notes by B. Singh), Tata Lecture Notes 57 (1977).

2. S. Abhyankar and T. T. Moh, Embeddings of the line in the plane, J. Reine Angew. Math. 276 (1975), 148-166.

3. R. Ganong, On plane curves with one place at infinity, Thesis, McGill University, Montréal, Canada, 1978.

4. $\ldots$, On plane curves with one place at infinity, J. Reine Angew. Math. 307/308 (1979), 173-193.

5. Kodaira dimension of embeddings of the line in the plane, J. Math. Kyoto Univ. 25 (1985), 649-657.

6. T. T. Moh, On the classification problem of embedded lines in characteristic $p$, Algebraic Geometry and Commutative Algebra in Honor of Masayoshi Nagata (Kinokuniya), 1987, 267-279.

Department of Mathematics, York University, Toronto, Ontario M3J 1P3 Canada 